Cite this: J. Mater. Chem. B, 2013, 1 3836

Received 1st May 2013

Accepted 19th June 2013

DOI: $10.1039 / c 3 t b 20630 k$

www.rsc.org/MaterialsB

\section{Eumelanin thin films: solution-processing, growth, and charge transport properties $\uparrow$}

\begin{abstract}
Julia Wünsche, ${ }^{a}$ Fabio Cicoira, ${ }^{b}$ Carlos F. O. Graeffc ${ }^{c}$ and Clara Santato*a
Eumelanin pigments show hydration-dependent conductivity, broad-band UV-vis absorption, and chelation of metal ions. Solution-processing of synthetic eumelanins opens new possibilities for the characterization of eumelanin in thin film form and its integration into bioelectronic devices. We investigate the effect of different synthesis routes and processing solvents on the growth, the morphology, and the chemical composition of eumelanin thin films using atomic force microscopy and X-ray photoelectron spectroscopy. We further characterize the films by transient electrical current measurements obtained at $50 \%$ to $90 \%$ relative humidity, relevant for bioelectronic applications. We show that the use of dimethyl sulfoxide is preferable over ammonia solution as processing solvent, yielding homogeneous films with surface roughnesses below $0.5 \mathrm{~nm}$ and a chemical composition in agreement with the eumelanin molecular structure. These eumelanin films grow in a quasi layer-bylayer mode, each layer being composed of nanoaggregates, 1-2 nm high, 10-30 nm large. The transient electrical measurements using a planar two-electrode device suggest that there are two contributions to the current, electronic and ionic, the latter being increasingly dominant at higher hydration, and point to the importance of time-dependent electrical characterization of eumelanin films.
\end{abstract}

\section{Introduction}

Melanins are biomacromolecules responsible for the pigmentation of many plants and animals. The biological functions of melanins, also present in the inner ear and the substantia nigra of the human brain, go far beyond coloration and include photoprotection, anti-oxidant behavior, and metal chelation. ${ }^{\mathbf{1 , 2}}$ Melanins are also intensively studied for their involvement in melanoma skin cancer and Parkinson's disease.,

Eumelanins, macromolecules mainly composed of 5,6dihydroxyindole (DHI) and 5,6-dihydroxyindole-2-carboxylic acid (DHICA) and their redox forms, are the most ubiquitous melanins in humans and the most studied by material scientists (Scheme 1). ${ }^{5}$ Back in the 1960-70s, eumelanin in form of pressed pellets was discovered to be conductive, photoconductive, and to show threshold switching. ${ }^{6-8}$ These properties and the broadband UV-vis absorption of eumelanin were explained mostly within the amorphous semiconductor

\footnotetext{
'Département de génie physique, Ecole Polytechnique de Montréal, CP 6079, Succursale Centre-Ville, Montréal, Québec H3C 3A7, Canada. E-mail: clara. santato@polymtl.ca

${ }^{b}$ Département de génie chimique, École Polytechnique de Montréal, CP 6079, Succursale Centre-Ville, Montréal, Québec H3C 3A7, Canada

${ }^{c}$ DF-FC, UNESP - Univ. Estadual Paulista, Av. Eng. Luiz Edmundo Carrijo Coube 1401, 17033-360 Bauru, Brazil

$\dagger$ Electronic supplementary information (ESI) available: AFM images of further spin-coated and drop cast eumelanin films and a long-time transient current measurement on Sigma melanin. See DOI: 10.1039/c3tb20630k
}

model.9,10 However, this interpretation has been challenged several times, especially considering the strong hydration dependence of the electrical properties of eumelanin. ${ }^{112}$ There is now growing evidence that protons play an important role in the charge carrier transport in eumelanin. ${ }^{\mathbf{1 3 - 1 5}}$ The potential for mixed ionic-electronic conduction combined with anti-oxidant, metal chelation properties, and the intrinsic biocompatibility of eumelanin make it an interesting candidate for organic bioelectronic applications.

Despite the recent research efforts, most fundamental properties of eumelanin such as its molecular structure and charge transport mechanism are still poorly understood. ${ }^{5}$ In particular, it is still a matter of debate whether eumelanin has an extended heteropolymeric structure or is composed of smaller oligomers, stacked in a graphite-like manner. ${ }^{\mathbf{1 6 , 1 7}}$ Eumelanin should furthermore be regarded as a mixture of chemically similar macromolecules rather than a well-defined chemical entity. ${ }^{\mathbf{1} 18}$ This chemical heterogeneity, the limited solubility of eumelanin and the amorphous character of materials based thereon make its characterization challenging. ${ }^{\mathbf{1 6}}$
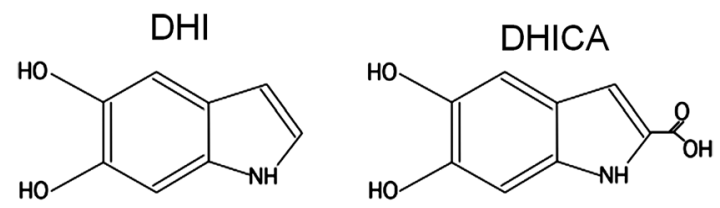

Scheme 1 Main building blocks of eumelanins: 5,6-dihydroxyindole (DHI) and 5,6-dihydroxyindole-2-carboxylic acid (DHICA). 
Due to difficulties in the extraction and purification of natural eumelanin without alteration of its molecular structure, different synthesis routes have been developed to prepare model eumelanins. ${ }^{19}$ Only recently, protocols for the solution processing of synthetic eumelanin thin films have been reported and the selection of potentially suitable solvents seems to be restricted to dimethyl sulfoxide (DMSO), ${ }^{20}$ dimethyl formamide ${ }^{20}$ and ammonia solutions. ${ }^{21}$ Therefore, there is an urgent need to investigate the effect of the synthesis route and processing conditions on the growth mechanism of eumelanin thin films and their final properties.

To explore the potential of eumelanin thin films for bioelectronics, which typically involves operation in aqueous environment, the charge transport properties of strongly hydrated eumelanin films need to be well characterized and understood. This effort would represent a first step towards the interfacing of eumelanin thin films with biologically relevant solutions. Studies of electrical properties of eumelanin thin films reported so far have been carried out in vacuum and ambient air (i.e. low film hydration) $)^{\mathbf{1 3 , 1 5 , 2 0 , 2 2 - 2 4}}$ or are limited to the determination of the film conductivity at $100 \%$ relative humidity (RH). ${ }^{\mathbf{2 1 , 2 5}}$ Studies on (partly) hydrated eumelanin films were commonly performed at high voltages $(\geq 10 \mathrm{~V}),{ }^{22,24,25}$ which might result in undesired currents due to water electrolysis, for example. Furthermore, $\mathrm{Au}$ or $\mathrm{Ag}$ were typically used as electrode materials in melanin thin film devices. ${ }^{13,15,21,22,25}$ However the use of these metals should be avoided, since they can electrochemically react with eumelanin, as we recently demonstrated. ${ }^{26}$

In this work, we investigate the morphology and chemical composition of solution-processed eumelanin thin films obtained with three different procedures: the auto-oxidation of L-dopa in aqueous suspension (Dopa melanin), ${ }^{27}$ a standard method reported in 1978; the auto-oxidation of $\mathrm{L}$-dopa in DMSO suspension (DMSO melanin), ${ }^{\mathbf{2 0}}$ which has been reported to yield a more homogeneous product with higher solubility; the oxidation of tyrosine with hydrogen peroxide, a product that is commercially available from Sigma Aldrich (Sigma melanin). Eumelanins films were spin-coated from DMSO and ammonia suspensions. Atomic force microscopy (AFM) and X-ray photoelectron spectroscopy (XPS) were employed to identify the most promising synthesis routes and processing solvents amongst those studied here, in terms of film morphology and chemical composition. AFM characterization of eumelanin films of various thicknesses also permitted to identify the growth mode of the solution-processed eumelanin films giving a simple explanation for the low surface roughness obtained even at film thicknesses of several hundreds of nanometers and lending support for the stacked oligomer picture of the eumelanin structure. Furthermore, we performed transient current measurements of eumelanin thin films at controlled humidity, ranging from $50 \%$ to $90 \%$, using Pt electrodes, upon application of electrical biases $\leq 1 \mathrm{~V}$. Such measurements supply important information on mixed ionic-electronic transport in eumelanin thin films, where the presence of mobile ions/protons has been revealed by infrared, electron spin resonance, and muon spin relaxation measurements. ${ }^{\mathbf{1 4 , 1 5}}$ Our measurements, at short and long timescales support the hypothesis of mixed ionic-electronic conduction and give new insights into the contribution of these two types of charge carriers at different hydration states.

\section{Experimental}

\section{Sample preparation}

Dopa melanin was synthesized according to the standard method reported in ref. 27. DMSO melanin was synthesized as described in ref. 20. DMSO melanin has to be stored under inert atmosphere, since it is prone to a slow degradation in air, significantly reducing its solubility. ${ }^{28}$ Sigma melanin and all other chemicals were purchased from Sigma Aldrich and used as received. The eumelanins were suspended in DMSO or ammonia solution (2:1 by volume, $\mathrm{NH}_{3(\mathrm{aq})}(28 \%)$ in deionized water), stirred for 30 minutes, and filtered. Concentrations of $0.3,3,15$, and $30 \mathrm{mg} \mathrm{ml}^{-1}$ were used to obtain films about 8, 15, 30 , and $50 \mathrm{~nm}$ thick by spin-coating. Film thicknesses were determined by AFM measurements on a scratch done on the film as well as by ellipsometry; the error associated to the measurement was of $25 \%$. The suspensions were spin-coated at $1000 \mathrm{rpm}$ for 2 minutes, followed by $4000 \mathrm{rpm}$ for $30 \mathrm{~s}$, on glass or thermally grown $\mathrm{SiO}_{2}$ on $\mathrm{Si}$ substrates $\left(\mathrm{SiO}_{2} / \mathrm{Si}\right)$. The substrates were pre-cleaned with isopropanol and acetone in an ultrasonic bath; $\mathrm{SiO}_{2}$ substrates were also exposed to UV-ozone for 15 min after wet cleaning. For electrical measurements, Pt electrodes (30 $\mathrm{nm}$ thick, including a $5 \mathrm{~nm}$ Ti adhesion layer) were deposited onto $\mathrm{Si} / \mathrm{SiO}_{2}$ wafers by e-beam evaporation and patterned by photolithography (planar configuration, interelectrode distance $L=10 \mu \mathrm{m}$, electrode width $W=7810 \mu \mathrm{m}$ ). Eumelanin films were deposited onto these patterned substrates using the same experimental conditions as described above.

\section{Sample characterization}

AFM measurements were taken with a Dimension 3100 (Digital Instruments) with Si probes (tip radius $<10 \mathrm{~nm}$, spring constant 20-100 $\mathrm{N} \mathrm{m}^{-1}$ ) in tapping mode. Images were analysed with Nanoscope Analysis from Bruker. The root mean square roughness was averaged over 3 images of the same sample.

XPS measurements were taken with an ESCALAB 3 MKII from VG Scienta with a $\mathrm{Mg} \mathrm{K} \alpha$ source. Measurements were taken on $30 \mathrm{~nm}$ thick films. The contribution of the $\mathrm{SiO}_{2}$ substrate was subtracted.

Electrical measurements were performed in air, in a chamber with controlled relative humidity. The Pt electrodes were contacted with micromanipulated tungsten probes. A software-controlled source/measure unit (Agilent B2902A) was used to apply voltage pulses between 0.2 and $1 \mathrm{~V}$ to a $30 \mathrm{~nm}$ thick Sigma melanin film and to record the current-time characteristics. After each pulse the voltage was set to $0 \mathrm{~V}$ for $1000 \mathrm{~s}$ to allow the sample to equilibrate. For verification, the backward current was recorded during these phases. The first measurement was taken at $90 \% \mathrm{RH}$ and the humidity was then decreased in steps of $10 \%$ for subsequent measurements. The sample was left for at least $24 \mathrm{~h}$ at constant humidity to 
equilibrate hydration before each measurement. A different device on the same substrate was used for each of the relative humidity states. Conductivity values were determined at an electrical bias $V=0.6 \mathrm{~V}$ by calculating $I(t) L /(V W d)$.

\section{Results and discussion}

\section{Thin film processing}

Fig. 1 shows $10 \mu \mathrm{m} \times 10 \mu \mathrm{m}$ AFM images of $30 \mathrm{~nm}$ thick films of Dopa melanin, DMSO melanin, and Sigma melanin spin-coated from DMSO and $\mathrm{NH}_{3(\mathrm{aq})}$ suspensions. The film root mean square roughness $(\mathrm{Rq})$ was only $0.3-0.4 \mathrm{~nm}$ for films deposited from DMSO and 0.4-0.5 $\mathrm{nm}$ for films deposited from $\mathrm{NH}_{3(\mathrm{aq})}$, with the exception of Dopa melanin, which tended to yield films decorated with larger aggregates, especially when deposited from $\mathrm{NH}_{3(\mathrm{aq})}$. Except for the latter case, AFM images show that homogeneous and smooth films can be deposited using the eumelanin-solvent combinations studied here. The slightly lower surface roughness obtained with DMSO might be due to the lower volatility of DMSO, which gives eumelanin molecules/ aggregates more time to arrange. The morphology is very similar for all these films, especially on a $1 \mu \mathrm{m} \times 1 \mu \mathrm{m}$ scale (Fig. S1†).

Interestingly, the similar film morphologies shown in Fig. 1 and $\mathrm{S} 1 \dagger$, correspond to distinct chemical compositions, as revealed by XPS measurements (Table 1). While Dopa and Sigma melanin deposited from DMSO suspension have a $\mathrm{C} / \mathrm{N} / \mathrm{O}$ content approximately within the expected range for a macromolecule consisting of DHI and DHICA, DMSO melanin has a non-negligible concentration of $\mathrm{S}$ and higher $\mathrm{C} / \mathrm{N}$ and $\mathrm{O} / \mathrm{N}$ ratios than the other two eumelanins. XPS data also reveal that processing eumelanin films in $\mathrm{NH}_{3(\mathrm{aq})}$ significantly increases the $\mathrm{N}$ content of the film and likely alters the molecular structure of eumelanin. The $\mathrm{C} / \mathrm{N}$ and $\mathrm{O} / \mathrm{N}$ ratios are more than a factor two lower for Sigma melanin films deposited from $\mathrm{NH}_{3(\mathrm{aq})}$ compared to films deposited from DMSO. The use of DMSO led to a slight increase in S content of less than $1 \mathrm{at} \%$. The increase in $\mathrm{S}$ concentration is not necessarily related to a modification of the eumelanin molecular structure but might reflect residues of the low volatile solvent DMSO.

Although a complete structural picture of eumelanin synthesis products is usually obscured by the heterogeneous and insoluble character of these pigments, it is well known from numerous studies that different precursors and oxidation conditions during synthesis lead to eumelanins differing in macromolecular structure, DHI/DHICA ratio, and the presence of different monomers. ${ }^{19,29}$ For example, it has been recently reported that DMSO melanin contains $-\mathrm{SO}_{2} \mathrm{CH}_{3}$ groups bound to the phenolic hydroxyl groups, which is consistent with our results. ${ }^{28}$ The effect of the processing solvent on the chemical composition of eumelanin films has been poorly investigated. Our results suggest that the use of Dopa and Sigma melanin deposited from DMSO suspensions yields preferentially films of a macromolecular material resembling the eumelanin pigment. The use of DMSO melanin or $\mathrm{NH}_{3(\mathrm{aq})}$ can yield smooth films, at the expense of a modified chemical composition. Because of the chemical modifications produced by the use of $\mathrm{NH}_{3(\mathrm{aq})}$ as processing solvent, we will not consider films deposited from $\mathrm{NH}_{3(\mathrm{aq})}$ suspensions any further in this work.

\section{Film growth}

To shed light on the growth of eumelanin thin films deposited from DMSO, spin-coated and drop cast films of various thicknesses $d$ were characterized by AFM. Spin-coated films with thicknesses of 30 and $50 \mathrm{~nm}$ consistently had smooth, homogeneous, and granular morphologies similar to those reported

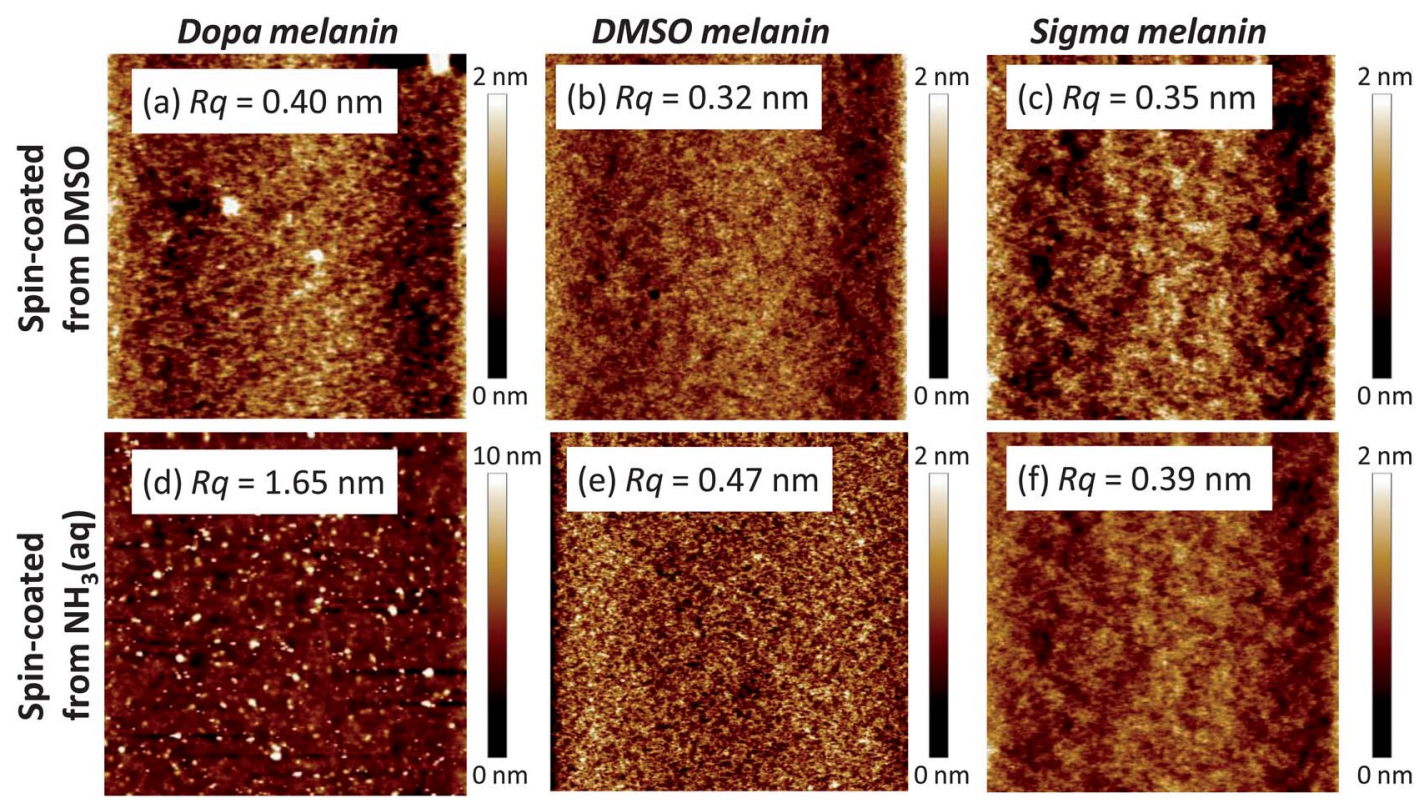

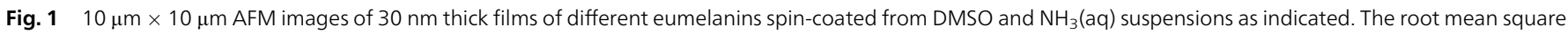
roughness $\mathrm{Rq}$ is indicated for each film. 


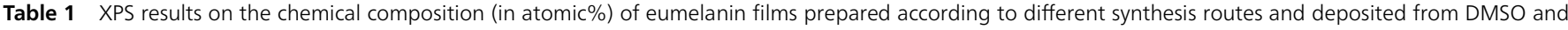

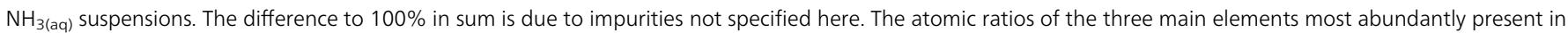

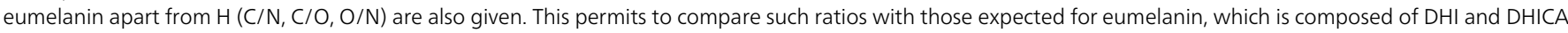
building blocks and their redox forms

\begin{tabular}{|c|c|c|c|c|c|c|c|c|}
\hline & $\begin{array}{l}\text { Dopa } \\
\text { melanin }\end{array}$ & $\begin{array}{l}\text { DMSO } \\
\text { melanin }\end{array}$ & $\begin{array}{l}\text { Sigma } \\
\text { melanin }\end{array}$ & $\begin{array}{l}\text { Dopa } \\
\text { melanin }\end{array}$ & $\begin{array}{l}\text { DMSO } \\
\text { melanin }\end{array}$ & $\begin{array}{l}\text { Sigma } \\
\text { melanin }\end{array}$ & Theoretical & Theoretical \\
\hline Solvent & DMSO & DMSO & DMSO & $\mathrm{NH}_{3}(\mathrm{aq})$ & $\mathrm{NH}_{3}(\mathrm{aq})$ & $\mathrm{NH}_{3}(\mathrm{aq})$ & DHI & DHICA \\
\hline C1s & 67.7 & 69.9 & 66.3 & 60.9 & 68.8 & 62.9 & - & - \\
\hline O1s & 22.9 & 22.4 & 26.1 & 22.9 & 21.0 & 22.4 & - & - \\
\hline N1s & 9.1 & 5.4 & 6.8 & 15.2 & 8.6 & 14.1 & - & - \\
\hline S2p & 0.2 & 1.9 & 0.5 & 0.1 & 1.1 & 0.3 & - & - \\
\hline $\mathrm{C} / \mathrm{N}$ & 7.4 & 12.9 & 9.8 & 4.0 & 8.0 & 4.4 & 8 & 9 \\
\hline $\mathrm{C} / \mathrm{O}$ & 3.0 & 3.1 & 2.5 & 2.7 & 3.3 & 2.8 & 4 & 2.25 \\
\hline $\mathrm{O} / \mathrm{N}$ & 2.5 & 4.1 & 3.8 & 1.5 & 2.4 & 1.6 & 2 & 4 \\
\hline
\end{tabular}

in Fig. 2 and $\mathrm{S} 1 . \dagger$ Besides that, most images on drop cast films $(d=0.1-1 \mu \mathrm{m})$ show comparably smooth surfaces (Fig. S2 $\dagger$ ).

In contrast, films with a thickness of $15 \mathrm{~nm}$ thick and less, had a more complex surface topography featuring flat islands at different stages of coalescence. Fig. 3a-c shows these islands for an $8 \mathrm{~nm}$ thick film of Sigma melanin. Similar morphologies were observed also for spin-coated DMSO melanin films $(d=15 \mathrm{~nm}, 8$ $\mathrm{nm}$, and less, Fig. S3 $\dagger$ ) and for several drop-cast samples, including DMSO melanin (Fig. 3d-f) and Dopa melanin (Fig. S4 $\dagger$ ). These flat islands observed at different film thicknesses indicate a quasi layer-by-layer growth mode and explain the low surface roughness observed for films with thicknesses of several hundreds of nanometers.

The height of the islands, typically 1-2 nm, is consistent with the height suggested for the fundamental nanoaggregate of eumelanin, composed of 3-6 stacked oligomers. ${ }^{16,30}$ High-resolution AFM images indicate that islands are made-up of diskshaped nanoaggregates, with a lateral extension of 10-30 nm (Fig. 3b; occasionally larger as for the drop cast film, Fig. 3e). Nanoaggregates of such width have been observed before ${ }^{21,31}$ and indicate either an edge-to-edge aggregation of the eumelanin fundamental nanoaggregate (with a width of about $2 \mathrm{~nm})^{\mathbf{1 6 , 3 0 , 3 2}}$ or oligomers with a relatively large lateral extension. The observation of these disk-shaped nanoaggregates for films

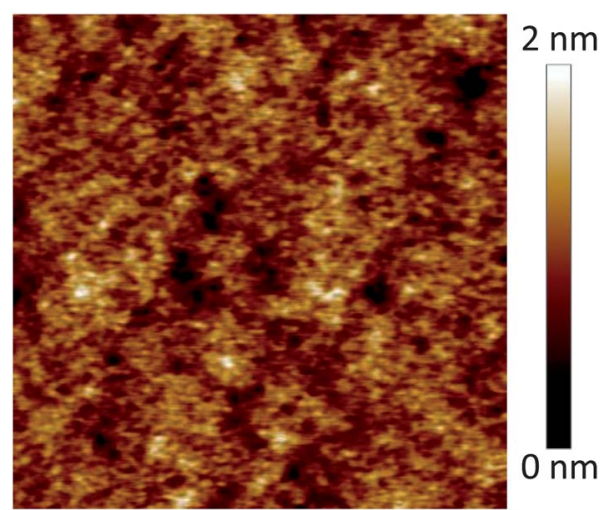

Fig. $21 \mu \mathrm{m} \times 1 \mu \mathrm{m}$ AFM image of a $30 \mathrm{~nm}$ thick film of Sigma melanin spincoated from DMSO. of Dopa, DMSO, and Sigma melanin with different thicknesses suggest that they are very stable and likely already present in the DMSO suspension (for this reason we avoided the word 'solution' in this work). It is possible that covalent bonds are formed between the stacked oligomers as suggested by Meng and Kaxiras, ${ }^{32}$ which would explain their insoluble character.

Eumelanin is known to aggregate in a hierarchical way forming structures with height and width up to several hundreds of nanometers. ${ }^{16,31}$ Our results suggest that DMSO stabilizes nanoaggregates of a height of about 1-2 $\mathrm{nm}$ and a width of 10-30 nm, preventing their further aggregation, and that their deposition in a quasi layer-by-layer manner allows the growth of homogeneous films with roughness below $0.5 \mathrm{~nm}$.

\section{Charge transport properties}

Fig. 4a shows the electrical response of eumelanin films (Sigma melanin deposited from DMSO) to $0.2 \mathrm{~V}$ to $1 \mathrm{~V}$ pulses, $3000 \mathrm{~s}$ long, in air, at different relative humidity values (RH). The first measurement was taken at high film hydration (90\% $\mathrm{RH})$ and the humidity was decreased for subsequent measurements. This procedure ensures that only the content of the weakly bound water is varied in the film, since the strongly bound water can be removed only by heating. ${ }^{5}$ It thus permits to disentangle the effect of the weakly bound water from the effect of the strongly bound water. The electrical current is dominated by a fast decreasing transient current within the first 100-500 s, depending on sample hydration. The fast decrease is followed by a much slower, nearly exponential decrease over several thousands of seconds. $t$ Longer measurements performed at 90\% RH showed that the current decreases more and more slowly without reaching a steady state (Fig. S5 $\dagger$ ). This behavior points to several contributions to the electrical current. We attribute the initial decrease of the current to the presence of ionic currents that are blocked at the Pt electrodes and persist until the electrochemical potential of mobile ions is equal throughout the eumelanin film. This interpretation is based also on previous reports on mobile protons in solid state

$\ddagger$ The temporary increase of current visible in some of the curves is due to external noise that could not be completely avoided. 

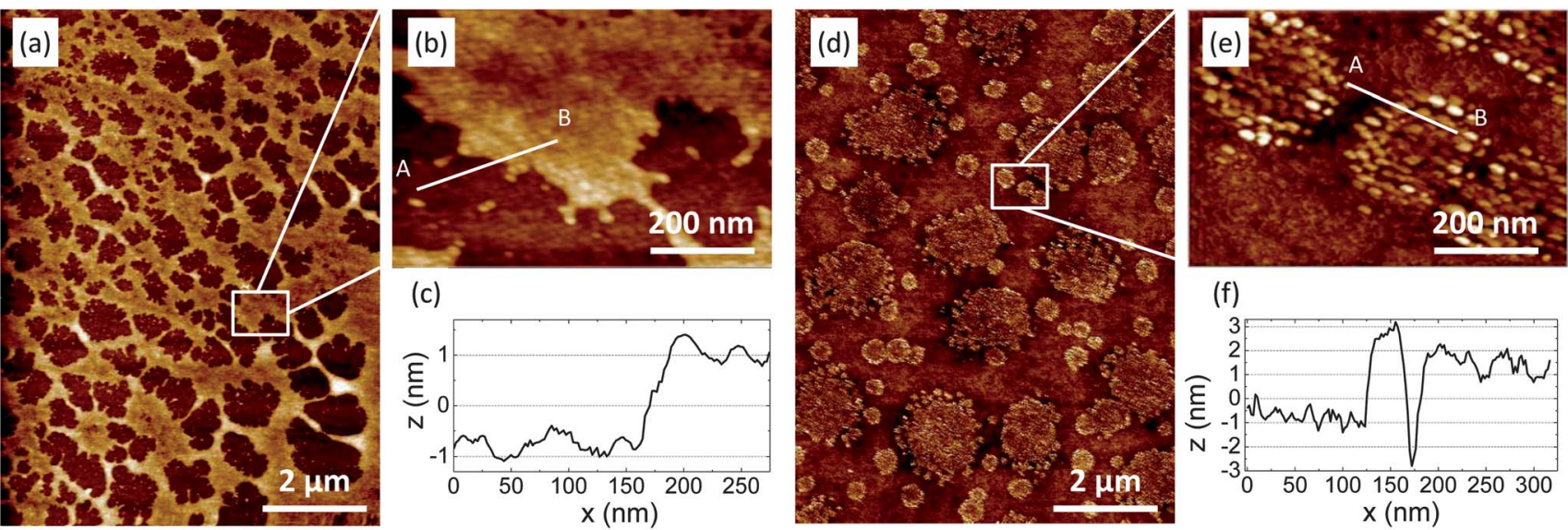

(f)

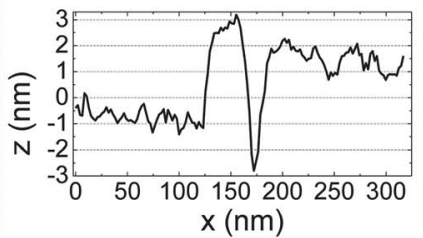

Fig. 3 AFM images of an about $8 \mathrm{~nm}$ thick film of Sigma melanin spin-coated from DMSO suspension on $\mathrm{SiO}_{2}((\mathrm{a})$ and (b), $z$-scale $6 \mathrm{~nm})$ and of drop-cast film of DMSO melanin on glass (thickness $>100 \mathrm{~nm}$ ), also using DMSO as a solvent ((d) and (e), z-scale $8 \mathrm{~nm})$. (c) and (f) are sections along the lines indicated in the images (b) and (e), respectively. Point A corresponds to $x=0, B$ to $x=275 \mathrm{~nm}$ and $325 \mathrm{~nm}$, respectively.
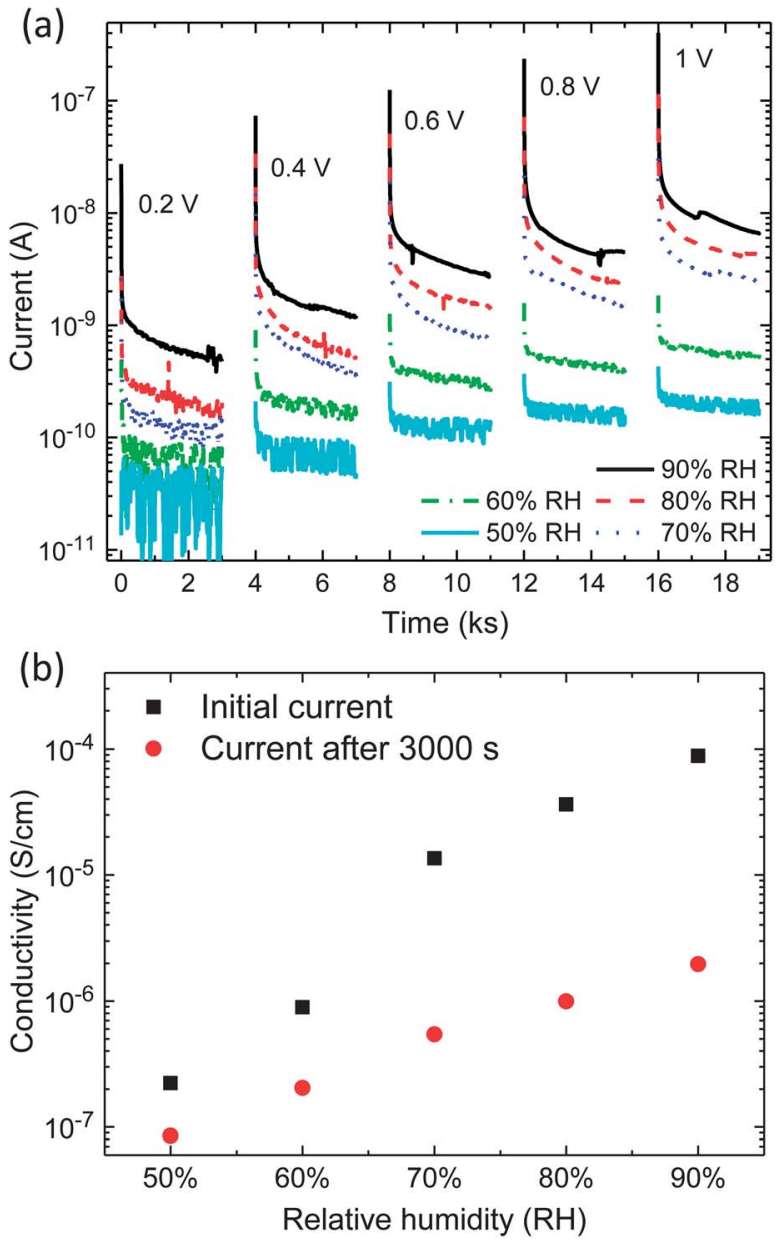

Fig. 4 (a) Transient current measurement of Sigma melanin film for $3000 \mathrm{~s}$ long voltage pulses from 0.2 to $1 \mathrm{~V}$ at relative humidity $(\mathrm{RH})$ varied between $90 \%$ and $50 \%$. Zero voltage was applied during the $1000 \mathrm{~s}$ between subsequent voltage pulses. The curves have been smoothened to reduce spikes caused by external noise. (b) Effective conductivity derived from the electrical current at $0.6 \mathrm{~V}$ at $0 \mathrm{~s}$ and $3000 \mathrm{~s}$ as a function of relative humidity. eumelanin..$^{12,14,15}$ The slowly decreasing current at longer time scales could be either due to a slower component of the ionic current as well as to electrochemical reactions or electronic current. The persistence of this current even after many hours of biasing and its presence at applied electrical biases as low as $0.2 \mathrm{~V}$ suggest that this current is of electronic type.

The well-known hydration dependence of eumelanin conductivity is clearly indicated in our current measurements. Both the ionic and electronic components of the current increase with the amount of weakly bound water. This is in agreement with the recent study of Mostert et al., ${ }^{14}$ which describes an increase in both mobile proton and extrinsic (semiquinone) free radical contents with hydration. We calculated, from the electrical currents at $V=0.6 \mathrm{~V}$ at $0 \mathrm{~s}$ and after $3000 \mathrm{~s}$, an apparent effective conductivity, plotted over the relative humidity to facilitate discussion and comparison with literature (Fig. 4b). The initial current at $90 \% \mathrm{RH}$ is more than 400 times larger with respect to the initial current at $50 \% \mathrm{RH}$, while the current after $3000 \mathrm{~s}$ is only about 20 times larger at $90 \% \mathrm{RH}$ with respect to $50 \% \mathrm{RH}$. This suggests that the ionic contribution to the electrical current is more important with increasing sample hydration and that neglecting the timedependence of the electrical current in eumelanin films interfaced with ion-blocking metal electrodes can easily lead to inconsistencies in DC conductivity. As a function of the time of biasing before reading the current, the conductivity might vary up to almost two orders of magnitudes. Conductivity values reported for spin-coated eumelanin films at 100\% $\mathrm{RH}(2.5 \times$ $10^{-5}$ and $\left.7 \times 10^{-5} \mathrm{~S} \mathrm{~cm}^{-1}\right)^{21,25}$ lie between those that could be obtained at short and long timescales according to our results when extrapolated to $100 \%$ RH. $§$

It should be noted here that ionic and electronic contributions to the electrical current cannot be considered as independent. The ionic current inevitably affects the electronic

$\S$ The extracted conductivity might also be affected by a number of other factors such as electrochemical reactions at the electrode-eumelanin interface, the presence of ionic impurities, and film morphology. 
current. On one hand, the ion accumulation close to the electrodes can facilitate electron and hole injection from the metal electrodes in the eumelanin films. ${ }^{33}$ On the other hand, the electric field created by these space charges is opposed to the external electric field and thus reduces the driving force for electronic transport within the eumelanin film. The local concentration of mobile protons and free radicals is furthermore coupled by the hydration-dependent comproportionation reaction. ${ }^{14}$ However, not all radicals and protons are necessarily mobile. Due to this complexity, further efforts using complementary experimental techniques and theoretical modeling are required to quantify the relative contribution of ionic and electronic conduction to the charge transport properties of eumelanin and to unravel the interplay of these two types of charge carriers in eumelanin thin films.

\section{Conclusions}

We investigated different combinations of synthesis routes and processing solvents for the fabrication of eumelanin thin films with respect to film growth, morphology, and chemical composition. We show that the use of Dopa or Sigma melanin deposited from DMSO is preferential for the fabrication of smooth eumelanin thin films with a chemical composition corresponding to eumelanin macromolecules. These nanoaggregates assemble in a quasi layer-by-layer growth mode, yielding films with surface roughness below $0.5 \mathrm{~nm}$. This observation supports the stacked-oligomer model, according to which eumelanin is composed of aggregated stacks of small oligomers rather than being an extended heteropolymer. We also investigated the time- and hydration- dependence of electrical currents in eumelanin thin films interfaced with Pt electrodes. Our results suggest two contributions to the electrical current, both increasing with the weakly absorbed water content of the sample. These contributions can be interpreted as ionic and electronic currents in agreement with results observed in electron spin resonance and muon relaxation studies recently published. ${ }^{\mathbf{1 4}}$ The ionic current became increasingly dominant at higher sample hydration. Our results represent a contribution towards the discovery of the factors governing charge transport in eumelanin and indicate the need for time-dependent electrical measurements. Further efforts using a combination of different characterization techniques and modelling are ongoing to gain a deeper understanding of charge transport in eumelanin films.

\section{Acknowledgements}

The authors are grateful to P. Moraille, J.-P. Lévesque, J. Bouchard, and Y. Drolet for technical support, to J. Lefebvre and S. Poulin for XPS measurements, to Professors A. Pezzella and M. Rolandi for scientific advice. J. W. is grateful to H. Guerboukha, P. Léger, and G. Taillon for part of the AFM measurements. Part of this work was carried out at the Central Facilities of École Polytechnique/Université de Montréal. J. W. is grateful to NSERC for financial support through a Vanier Canada Graduate Scholarship. C. S. acknowledges financial support by FQRNT
(Équipe) and by the Québec MDEIE-PSR-SIIRI. FC acknowledges financial support by NSERC (Discovery grant).

\section{Notes and references}

1 G. Prota, Melanins and melanogenesis, Academic Press, San Diego, 1992.

2 P. A. Riley, Int. J. Biochem. Cell Biol., 1997, 29, 1235.

3 H. Fedorow, F. Tribl, G. Halliday, M. Gerlach, P. Riederer and K. L. Double, Prog. Neurobiol., 2005, 75, 109.

4 H. Z. Hill, W. Li, P. Xin and D. L. Mitchell, Pigm. Cell Res., 1997, 10, 158.

5 P. Meredith and T. Sarna, Pigm. Cell Res., 2006, 19, 572.

6 J. McGinness, P. Corry and P. Proctor, Science, 1974, 183, 853.

7 P. Crippa, V. Cristofoletti and N. Romeo, Biochim. Biophys. Acta, Gen. Subj., 1978, 538, 164.

8 A. M. Potts and P. Chit Au, Agressologie, 1968, 9, 225.

9 J. E. McGinness, Science, 1972, 177, 896.

10 M. M. Jastrzebska, H. Isotalo, J. Paloheimo and H. Stubb, J. Biomater. Sci., Polym. Ed., 1995, 7, 577.

11 A. B. Mostert, B. J. Powell, I. R. Gentle and P. Meredith, Appl. Phys. Lett., 2012, 100, 093701.

12 M. R. Powell and B. Rosenberg, J. Bioenerg., 1970, 1, 493.

13 M. Ambrico, P. F. Ambrico, A. Cardone, T. Ligonzo, S. R. Cicco, R. Di Mundo, V. Augelli and G. M. Farinola, Adv. Mater., 2011, 23, 3332.

14 A. B. Mostert, B. J. Powell, F. L. Pratt, G. R. Hanson, T. Sarna, I. R. Gentle and P. Meredith, Proc. Natl. Acad. Sci. U. S. A., 2012, 109, 8943.

15 P. J. Gonçalves, O. B. Filho and C. F. O. Graeff, J. Appl. Phys., 2006, 99, 104701.

16 M. D'Ischia, A. Napolitano, A. Pezzella, P. Meredith and T. Sarna, Angew. Chem., Int. Ed., 2009, 48, 3914.

17 S. Reale, M. Crucianelli, A. Pezzella, M. D'Ischia and F. De Angelis, J. Mass Spectrom., 2012, 47, 49.

18 P. Meredith, B. J. Powell, J. Riesz, S. P. Nighswander-Rempel, M. R. Pederson and E. G. Moore, Soft Matter, 2006, 2, 37.

19 A. Pezzella and J. Wünsche, in Organic Electronics: Emerging Concepts and Technologies, ed. F. Cicoira and C. Santato, Wiley-VCH, 2013.

20 S. N. Dezidério, C. A. Brunello, M. I. N. da Silva, M. A. Cotta and C. F. O. Graeff, J. Non-Cryst. Solids, 2004, 338-340, 634.

21 J. P. Bothma, J. de Boor, U. Divakar, P. E. Schwenn and P. Meredith, Adv. Mater., 2008, 20, 3539.

22 F. Bloisi, A. Pezzella, M. Barra, M. Alfè, F. Chiarella, A. Cassinese and L. Vicari, Appl. Phys. A: Mater. Sci. Process., 2011, 105, 619.

23 M. Abbas, F. D'Amico, L. Morresi, N. Pinto, M. Ficcadenti, R. Natali, L. Ottaviano, M. Passacantando, M. Cuccioloni, M. Angeletti and R. Gunnella, Eur. Phys. J. E: Soft Matter Biol.Phys., 2009, 28, 285.

24 S. Subianto, G. Will and P. Meredith, Polymer, 2005, 46, 11505.

25 C. J. Bettinger, J. P. Bruggeman, A. Misra, J. T. Borenstein and R. Langer, Biomaterials, 2009, 30, 3050. 
26 J. Wünsche, L. Cardenas, F. Rosei, F. Cicoira, R. Gauvin, C. F. O. Graeff, S. Poulin, A. Pezzella and C. Santato, Adv. Funct. Mater., 2013, DOI: 10.1002/adfm.201300715.

27 C. C. Felix, J. S. Hyde, T. Sarna and R. C. Sealy, J. Am. Chem. Soc., 1978, 100, 3922.

28 E. S. Bronze-Uhle, A. Batagin-Neto, P. H. P. Xavier, N. I. Fernandes, E. R. de Azevedo and C. F. O. Graeff, J. Mol. Struct., 2013, 1047, 102.

29 M. R. Chedekel, A. B. Ahene and L. Zeise, Pigm. Cell Res., 1992, 5, 240.
30 G. Zajac, J. Gallas, J. Cheng, M. Eisner, S. Moss and A. Alvaradoswaisgood, Biochim. Biophys. Acta, Gen. Subj., 1994, 1199, 271.

31 C. M. R. Clancy, J. B. Nofsinger, R. K. Hanks and J. D. Simon, J. Phys. Chem. B, 2000, 104, 7871.

32 S. Meng and E. Kaxiras, Biophys. J., 2008, 94, 2095.

33 J. D. Slinker, J. A. DeFranco, M. J. Jaquith, W. R. Silveira, Y. Zhong, J. M. Moran-Mirabal, H. G. Craighead, H. D. Abruña, J. A. Marohn and G. G. Malliaras, Nat. Mater., 2007, 6, 894. 